\title{
1 Pituitary carcinoma: The University of Texas MD Anderson Cancer Center
}

\section{2 experience.}

Marta Penas-Prado, MD. Department of Neuro-oncology. MD Anderson Cancer Center. Houston, TX. marta.penas-

Carlos Kamiya-Matsuoka, MD. Department of Neuro-oncology. MD Anderson Cancer Center. Houston, TX.

11 ckamiya@mdanderson.org

Paul D Brown, MD. Department of Radiation Oncology. Mayo Clinic, Rochester, MN. Brown.paul@mayo.edu

Disclosures: None of the above authors declare any funding or disclosures.

20 Keywords: pituitary carcinoma, neuroendocrine tumors, temozolomide, chemoradiation. 


\section{Pituitary carcinoma: The University of Texas MD Anderson Cancer Center}

23 experience.

Santos-Pinheiro F, Penas-Prado M*, Kamiya-Matsuoka C*, Waguespack SG, Mahajan A, Brown PD, Shah KB, Fuller GN, McCutcheon IE. *both authors contributed equally.

\section{Abstract:}

Background: Pituitary carcinoma (PC) is an aggressive neuroendocrine tumor diagnosed when a pituitary adenoma (PA) becomes metastatic. PCs are typically resistant to therapy and frequently recur. Recently, treatment with temozolomide (TMZ) has shown promising results, although the lack of prospective trials limits accurate assessment. Methods: We describe a single-center experience in managing PC over a 22year period and review previously published PC series. Results: 17 patients were identified. Median age at PC diagnosis was 44 years (range 16-82), and the median PA-to-PC conversion time was 5 years (range 1-29). Median follow-up was 28 months (range 8-158) with 7 deaths. Most PC were hormone-positive based on immunohistochemistry ( $n=12)$ : ACTH $(n=5), \operatorname{PRL}(n=4), \operatorname{LH} / \mathrm{FSH}(n=2), \mathrm{GH}(n=1)$. All patients underwent at least one resection and one course of radiation after PC diagnosis. Immunohistochemistry showed high Ki-67 labeling index (>3\%) in 10/15 cases. Eight patients (47\%) had metastases only to the CNS, and 6 (35\%) had combined CNS and systemic metastases. The most commonly used chemotherapy was TMZ, and TMZ-based therapy was associated with the longest period of disease control in 12 (71\%) cases, as well as the longest period from PC diagnosis to first progression in 8 (47\%) cases. The 2, 3 and 5-year survival rate of the entire cohort was $71 \%, 59 \%$ and $35 \%$, respectively. All patients surviving $>5$ years were treated with TMZ-based therapy. Conclusions: PC treatment requires a multidisciplinary 
approach and multimodality therapy including surgery, radiation and chemotherapy. TMZ-based therapy was associated with higher survival rates and longer disease control.

\section{Precis}

We describe 17 PC patients who were diagnosed and treated at MDACC over a 22-year period. We have found that TMZ-based therapy correlated with longer disease control and higher survival rate.

\section{Introduction:}

Pituitary carcinoma (PC) is a rare and aggressive neuroendocrine tumor (NET) accounting for approximately $0.1 \%$ of all pituitary neoplasms. ${ }^{1}$ The diagnosis is established after a pituitary adenoma (PA) becomes metastatic. ${ }^{2-4}$ Although metastases along the neuro-axis are more frequently observed, spread outside the CNS is also seen. The typical PA to PC latency period is several years, and little is known of the drivers for dissemination. Although p53 expression and

Ki-67 labeling index correlate well with the degree of peritumoral invasion and aggressive behavior in PA, no specific histological or molecular markers are required to diagnose PC. ${ }^{3,5}$ The management is challenging and a combination of surgical resection and/or radiation therapy is typically recommended, with chemotherapy often used when surgery and radiation are not possible or were previously unsuccessful. Nevertheless, response to standard treatment is usually transient and PC recurrence is common, with a reported median overall survival (OS) of 1 year. ${ }^{1,6}$ Improved outcome has been recently reported with the use of chemotherapy in 
64 recurrent pituitary neuroendocrine tumors, ${ }^{1,7}$ especially temozolomide $(\mathrm{TMZ})^{8-18}$ as a single

65 agent, in combination with capecitabine ${ }^{19}$ or concurrently with radiotherapy ${ }^{20,21}$. Recent

66 practice guidelines for the management of aggressive pituitary tumors and PC have

67 recommended temozolomide as first line chemotherapy. ${ }^{22}$ Here we summarize a 22 -year

68 single-center experience treating newly diagnosed and recurrent PC, with an emphasis on the

69 use of TMZ-based therapy.

71 Methods:

72 We conducted a retrospective review of all adult PC patients included in the University of Texas

73 MD Anderson Cancer Center (MDACC) institutional databases from October 1, 1994 through

74 January 31, 2017 under a protocol with waiver of consent approved by the Institutional Review

75 Board. All patients had undergone a biopsy or surgical resection of a pituitary mass and/or

76 metastatic disease. Patients' demographic and clinical characteristics, treatments and outcome

77 were reviewed. The diagnosis of PC was based on radiographic studies demonstrating

78 metastatic dissemination and confirmed in equivocal cases by diagnostic biopsy or resection of

79 a CNS or systemic lesion. Tumors were classified based on immunohistochemistry (IHC) findings

80 and blood hormone levels (when available) into somatotroph (GH-PC), lactotroph (PRL-PC),

81 corticotroph (ACTH-PC), thyrotroph (TSH-PC), gonadotroph (LH/FSH-PC) and null cell (NC-PC)

82 subtypes. $^{23}$ Since each PC patient received several lines of treatment (surgery, XRT,

83 chemotherapy), we calculated the period of disease control achieved by each treatment

84 modality. Disease control was defined as the presence of stable or decreased tumor burden in 
85 the primary sellar/locally invasive tumor and at all metastatic locations. We then determined

86 which treatment modality was associated with the longest period of disease control. We

87 calculated overall survival from the initial diagnosis of PC to death. PC progression was defined

88 based on radiographic evidence of new or interval growth of metastatic lesions or of the

89 primary tumor itself. Death was confirmed by review of medical records and/or death

90 certificate.

91

92 Results:

\section{- Demographics}

94 A total of 17 patients with PC were seen over the study period. There was no gender

95 predilection (Male:Female $=9: 8$, Table 1 ). The median age at diagnosis of PC was 44 years

96 (range 16-82 years). Thirteen patients with PC were diagnosed and treated at MDACC, while 4

97 had the PC diagnosis confirmed in our institution but were treated elsewhere. Of note, 3 of the

98 study subjects have already been presented in previous publications. ${ }^{21,24}$

100 The median PA to PC time to transformation (TTT) was 5 years (range 1-29 years, Table 1). One

101 patient was diagnosed simultaneously with PA and metastatic disease (de novo PC). Among the

102 IHC hormone-positive subtypes, ACTH-PC had the longest median TTT (5 years, range 2-29),

103 followed by PRL-PC (4 years, range 2-12 years), LH/FSH-PC (3 years, range 2-4 years) and GH-PC

104 (2 years). The median TTT of NC-PC was 9 years (range 1-15 years). 
105 All patients were symptomatic at the time of diagnosis of PC and clinical presentation most 106 commonly involved neurological symptoms such as headaches ( $n=9[53 \%]$ ) and visual 107 impairment $(n=5[30 \%])$. Other symptoms such as facial pain, focal weakness, increased thirst, 108 and weight gain were also reported.

110 Pituitary tumors were classified based on IHC findings into somatotroph (GH-PC), lactotroph 111 (PRL-PC), corticotroph (ACTH-PC), gonadotroph (LH/FSH-PC) and null cell (NC-PC). IHC was not 112 available in 2 cases which were therefore classified based on blood hormone levels (1 PRL-PC 113 and $1 \mathrm{GH}-\mathrm{PC}$ ); there were no cases of TSH-PC, plurihormonal, or double tumors. The majority of 114 PC (n=12) were hormone-positive: ACTH-PC (n=5), PRL-PC $(n=4)$, LH/FSH-PC $(n=2)$ and GH-PC $115(n=1)$. Five patients were NC-PC (Table 1). The one GH-PC patient had clinical signs of 116 acromegaly. Three out of the 5 ACTH-PC patients developed Cushing syndrome.

117 Either before or after PC diagnosis was suspected based on imaging findings of metastatic 118 lesions, all patients underwent at least one surgical resection of the pituitary tumor (mean of 119 2.3, range 1-6). IHC studies (15 sellar tumor samples available) showed a median Ki-67 index of $12011 \%$ (range 1-40\%); high Ki-67 labeling index (>3\%) was present in 10/15 (67\%) sellar tumor 121 samples. Staining for mutant P53 was positive in 2 out of 9 tumor samples tested. Assessment 122 for MGMT expression was not available in this cohort as it was not routinely done as part of 123 clinical care.

124 Molecular studies via PCR-based next generation sequencing of tumor samples (primary and/or 125 metastatic site) was done in 5 patients, and a hypermutator phenotype was present in 3 of 
126

127

128

129

130

131

132

133

134

135

136

137

138

139

140

141

142

143

144

145

146

147

them ( $A C T H-P C=2 ; N C-P C=1)$ : in one $A C T H-P C$ patient, an initial 46-gene panel was negative, but when a 400-gene panel was used, 20 potentially actionable gene mutations were identified (ATM, GNAS, APC, CCNE1, CSF1R, EML4, ERBB3, ERBB4, FBXW7, FGFR4, IL7R, JAK1, MLL, MLL2, MLL3, NF2, NTRK3, PDGFRB, PTPN11, TOP1) along with 53 other gene mutations identified in a tumor sample from tumor extension into the orbit. The other ACTH-PC patient had 1 potentially actionable mutation (CREBBP splice site 2086_2113+49del77) along with another 20 gene variations of unknown significance identified in progressive sellar tumor. In the NC-PC patient, a metastatic tumor sample tested positive for MSH6; the MGMT methylation profile was negative. Among the two patients without hypermutator phenotype, a PRL-PC had no mutations identified in 147 genes tested in a metastatic tumor sample, while a NC-PC patient had a primary tumor sample positive for FGFR1 amplification.

\section{- Tumor location}

Prior to PC diagnosis, 15 out of 17 pituitary tumors were locally invasive (one case had no information available about tumor extension prior to PC diagnosis; one case was diagnosed simultaneously with a PA and a distant CNS lesion). The most common site of local invasion was the cavernous sinus $(n=8,47 \%)$ and sphenoid sinus $(n=8,47 \%)$. Involvement of optic structures (optic nerves, orbit, chiasm) was observed in $35 \%$ of the patients $(n=6)$, resulting in visual loss in 3 cases caused by compression of nerve rather than frank invasion. Most patients $(n=13)$ developed central nervous system (CNS) metastases (dural based, $n=13$; leptomeningeal, $n=3$ ) (Figure 1A, 1B). In eight patients (47\% of the entire cohort) metastasis was found only in the CNS, although the systemic work-up was not standardized in this cohort. Six (35\%) patients had concomitant CNS and extra-CNS metastases (bone, $n=3$; lymph nodes, $n=1$; other organ (liver, 
148 lung), $n=2$ ) (Figure 1B), with two of them having more than one location of extra-CNS 149 metastasis. Three (17\%) patients had extra-CNS metastases without any metastases within the 150 CNS (Table 1).

152 The majority of patients received multimodality therapy.

\subsection{Surgery}

154 The average number of surgical pituitary resections from the initial PA diagnosis was 1.8 per

155 patient (range 1-3). Seven patients (41\%), required a craniotomy for further resection after an

156 initial transsphenoidal sub-total resection of PA. The most common surgical techniques for

157 resection of pituitary tumor were endonasal transsphenoidal approach ( $n=14$ ) (including 1

158 endoscopic approach), followed by) pterional craniotomy $(n=10)$ and sublabial transsphenoidal

159 approach $(n=2)$. Other surgical approaches consisted of transfacial/ lateral rhinotomy and

160 fronto-orbito-zygomatic craniotomy (1 case each). Complications related to surgery occurred in

1612 patients and consisted of cerebrospinal fluid (CSF) leak following the fronto-orbito-zygomatic

162 approach requiring redo craniotomy and packing of the frontal sinus and sella, and

163 repositioning of a ventriculoperitoneal shunt (which was placed following prior surgical

164 resection of a PA that was causing hydrocephalus) following redo transsphenoidal approach for

165 subsequent pituitary tumor resection. Of note, 3 ACTH-PC patients underwent bilateral

166 adrenalectomy prior to PC diagnosis to control refractory Cushing's disease. 
168

169

170

171

172

173

174

175

176

177

178

179

180

181

182

183

184

185

186

187

188

Following PC diagnosis, $7(41 \%)$ patients were treated with radiotherapy to the sella $(n=5)$ or radiotherapy concurrent with $\mathrm{TMZ}(\mathrm{n}=2)$. Intensity-modulated radiation therapy (IMRT, total dose: $45-54 \mathrm{~Gy}$, divided in 25-30 fractions) was the most common radiotherapy modality in this setting. With regards to metastatic disease, a total of 13 foci were treated with radiotherapy (CNS metastases: $n=7$; extra-CNS metastases: $n=6$ ). IMRT was the most frequent technique $(n=10)$, followed by stereotactic radiosurgery (SRS, $n=4)$, craniospinal radiation (CSRT, $n=2$, both in the setting of leptomeningeal carcinomatosis) and intensity-modulated proton therapy (IMPT, $n=1)$.

\subsection{Chemotherapy}

TMZ was the most frequently prescribed chemotherapy in this series (Table 1), and it was used in all $\mathrm{PC}$ subtypes: $\mathrm{NC}=5 / 5 ; \mathrm{ACTH}=4 / 5 ; \mathrm{PRL}=3 / 4 ; \mathrm{LH} / \mathrm{FSH}=1 / 2 ; \mathrm{GH}=1 / 1 . \mathrm{TMZ}$ was used for newly diagnosed PC as monotherapy $(n=4)$, concurrently with radiotherapy $(n=2)$ and immediately following radiotherapy $(n=2)$. The most common TMZ scheduling and dosage was $150 \mathrm{mg} / \mathrm{m} 2$ for 5 days on a 28-day cycle, for 12 months $(n=6)$. TMZ was also used for recurrent PC in 7 patients; it was given as monotherapy $(n=5)$, combined with capecitabine $(n=2$; CAPTEM: capecitabine $1200 \mathrm{mg} / \mathrm{m} 2 /$ day in divided doses twice daily, days 1 to 14, and TMZ $150-200 \mathrm{mg} / \mathrm{m} 2 /$ day in divided doses twice daily, days 10 to 14 of a 28-day cycle, for a total of 6 cycles $)^{11}$, or concurrently with radiotherapy $(n=1 ; T M Z 75 \mathrm{mg} / \mathrm{m} 2$ daily for 42 days combined with radiation 5 days a week for 6 weeks). Four patients (one LH/FSH-PC, one ACTH-PC and two PRL-PC) were re-challenged with a TMZ-based therapy upon PC recurrence. One of them (LH/FSH-PC) was treated with TMZ upon PC diagnosis and had recurrence less than a year later; this patient then received concurrent TMZ and radiotherapy and died less than one year later 
190 (OS 5 years). The other 3 patients were treated with TMZ-based therapy upon first and

191 subsequent PC recurrences; all of them were initially treated with TMZ alone (which conferred

192 a median PFS of 1.5 years, range 1-2.5 years), and were then treated with CAPTEM, which

193 provided progression-free survival of less than 1 year.

194 TMZ therapy was overall well tolerated with anticipated side effects, and in none of the 195 patients was the therapy terminated or delayed due to hematological or non-hematological 196 adverse events. The most common adverse events attributed to TMZ were fatigue and nausea 197 (35\%), with no reported grade III-V toxicity, according to CTCAE v4.0 (2009).

198 Other chemotherapy regimens used for newly diagnosed PC were cisplatin (after concurrent 199 radiation and $\mathrm{TMZ}, \mathrm{n}=1)$, cisplatin plus etoposide $(\mathrm{n}=1)$, and cyplophosphamide in combination 200 with vincristine, doxorubicin and dacarbazine (CyVADIC, $n=1$ ). In the recurrent setting, 201 treatments included bevacizumab as a single agent $(n=3)$, bevacizumab plus irinotecan $(n=1)$, 202 bevacizumab plus pan-FGFR kinase inhibitor ( $n=1$, as part of a phase 1 clinical trial), 203 bevacizumab plus lomustine $(n=1)$, carboplatin plus vincristine $(n=1)$ and carboplatin plus 5 -FU $204(n=1)^{24}$

205 In this series, 3 patients were enrolled in 3 different phase 1 clinical trials testing: 1) tipifarnib 206 plus sorafenib, a farnesyltransferase inhibitor and a multi-tyrosine kinase inhibitor (VEGFR, 207 PDGFR, Raf), respectively; 2) pembrolizumab, a PD-1 inhibitor, and 3) BGJ398, a pan-FGFR 208 inhibitor. The outcome of these patients treated with experimental drugs is not discussed here. 
210 Progression after any given therapy, i.e., after first treatment for newly diagnosed PC and after

211 any treatment for subsequent progression, if applicable, occurred in 10 (59\%) patients, at a

212 median time of 18 months (range 0.33 - 13 years) and at a median occurrence of 1 (range 1-10

213 occurrences) per patient. Of the 7 patients without imaging evidence of recurrence during

214 follow up, 4 were diagnosed with PC less than 3.5 years prior to the end of the study and were

215 still alive, and 3 patients were deceased in less than one year from PC diagnosis. The cause of

216 death was unknown, but death due to progression of PC could not be excluded. Median time

217 from PC diagnosis to first PC recurrence was 9 months (range 3->42 months). Eight patients

218 (47\%) were treated with upfront TMZ or TMZ-based therapy (Table 1) and achieved a median

219 time from PC diagnosis to first PC recurrence of 30 months (range 5->42 months). Another 8

220 patients (47\%) were treated with upfront chemo- or radiotherapy without TMZ and achieved a

221 median time from PC diagnosis to first PC recurrence of 10 months (range 0.33- 13 years). One

222 patient (PRL-PC) received no treatment due to poor performance status at PC diagnosis (OS 8

223 months). One patient (NC-PC, initial metastatic site: cerebellum) achieved 13 years of stable

224 disease without upfront therapy and is now on TMZ following the $4^{\text {th }}$ recurrence of disease

225 (further metastatic sites: pons, lung and bone). Overall, TMZ-based therapy was associated with

226 the longest period of disease control in 12 (71\%) patients when used at diagnosis or recurrence

227 (median 21 months, range 4->42 months, Table 1). The median follow-up after PC diagnosis was

2282 years (range 1-13 years). The 2-, 3- and 5-yearsurvival rates for the entire cohort were $71 \%$

$229(n=12), 59 \%(n=10)$, and 35\% $(n=6)$, respectively. Of the patients who were alive at least 5 years

230 from diagnosis, 2 patients (one NC-PC, one ACTH-PC, both still alive) survived more than 10

231 years and all 5 had received treatment with TMZ-based therapy (Table 1). One refractory case is 
232 currently under treatment with the PD-1 inhibitor pembrolizumab as part of a phase 1 clinical

233 trial. Eighty percent of NC-PC were alive at last follow-up, in contrast with $58 \%$ of IHC hormone-

234 positive PC. There were 7 reported deaths by the end of the study period. Cause of death was

235 infectious etiology in 1 patient (pneumonia, while off temozolomide for one month) and 236 unknown in 6 patients.

\section{Discussion:}

239 Pituitary carcinoma (PC) is a rare and aggressive form of pituitary tumor for which limited

240 therapeutic guidelines exist. As no curative treatment has been established for PC to date, this

241 case series aimed to present our experience with the use of multiple modalities of treatment

242 over a prolonged period of follow-up (median, 2 years; range, 1-13 years). Our experience

243 suggests that a multimodality approach involving a combination of surgical resection,

244 radiotherapy and chemotherapy (particularly TMZ-based) correlates with better outcome (2-

245 year OS rate 71\%, 3-year OS rate 59\%, 5-year OS rate 35\%), compared with previous reports in

246 the literature (Table 2). ${ }^{1,6,7,21}$

247 According to the World Health Organization (WHO) classification of pituitary tumors $(2017)^{4}$,

248 the presence of metastatic disease in the CNS or systemically suffices to designate a pituitary

249 tumor as PC. However, this definition carries important limitations. This approach disregards

250 intrinsic histopathologic or molecular features of the pituitary tumor and relies exclusively on

251 the detection of metastasis. However, despite metastatic activity, the metastatic disease itself 
252 may remain devoid of typical malignant features (i.e. p53 expression, tumor invasion, high 253 proliferation rate).

254 The strategic location of the pituitary in relation to other intracranial structures (internal 255 carotid artery, dura, cavernous sinus) may facilitate the hematogenous, CSF and lymphatic 256 spread of cancerous cells into the neuraxis and extra-CNS locations. Yet, metastatic disease 257 occurs in only $0.1 \%$ of all PA cases and little is known of the biologic drivers for malignant 258 behavior in these tumors. The majority of patients in our series had CNS metastasis with 259 involvement of the meninges $(n=11)$, which raises the question of the mechanism of spread of 260 PC, either by CSF or hematogenously, given its close proximity to vascular structures as well as 261 the meninges lining the sella. Extra-CNS metastases involving vertebral bodies, long bones, 262 liver, lung, and lymph nodes were seen in over half the patients in our series.

263 The spectrum between PA and PC likely implies biologic differences beyond the presence of 264 metastases, although these are unknown at present. The 2017 WHO classification aimed to 265 improve tumor classification by removing the term "atypical PA" as a pituitary tumor subtype 266 (PA with increased proliferation rate [Ki-67 labeling index $>3 \%$ and increased mitotic figures], 267 p53 overexpression, local tumor invasion, and atypical morphologic features). However, the 268 new WHO classification still recommends the use of the term "aggressive PA" (invasion pattern, 269 rapid recurrence or resistant to therapy) to classify tumors deviating from a 'benign' behavior.

270 Although the terms 'aggressive' and 'invasive' have been used interchangeably in the literature, 271 such mixing blurs the distinctions among rapid tumor growth, invasion of adjacent structures, 272 metastasis to distant sites, and resistance to treatment. Such distinctions are necessary for a 273 biologically relevant definition of an "aggressive PA." Moreover, despite evidence of correlation 
274 between histologic proliferation markers and clinical aggressiveness in PA, no specific cutoff

275 was recommended for Ki-67 and mitotic index by the 2017 WHO classification, which does not

276 provide clear guidelines for classification or treatment of such tumors. In clinical practice,

277 however, the presence of these histologic markers is commonly incorporated in decisions about

278 frequency of surveillance and in discussions with patients about the potential risk for

279 recurrence.

280 In our series, the mean latency period from diagnosis of PA to PC transformation was 5 years

281 (range, 1-29 years), which is in accordance with the literature. ${ }^{1,6}$ Among the IHC hormone-

282 positive PC subtypes, GH- and LH/FSH-PC had the shortest latency period, while PRL-PC, ACTH-

283 PC, and NC-PC had the longest latency. However, given the small number of patients with each

284 subtype in our series, we cannot determine with certainty if this holds true in the entire

285 population of patients with PC.

286 The Ki-67 labeling index was assessed in the sellar tumor in the majority of our patients $(n=15)$

287 and, before the PC diagnosis was established, it was high (more than $3 \%$ ) in $67 \%$ of them. The

288 present series and review of the literature support that the vast majority of PC arise from

289 previously locally infiltrative tumors with high proliferation rate; however, a low Ki-67 does not

290 eliminate the risk of malignant transformation. Other pathologic biomarkers such as mutant

291 p53 expression, MGMT IHC or promoter methylation status, MSH6 IHC, and mutation profile

292 were not systematically tested in our series, although they may be of clinical relevance for

293 prediction of treatment response (i.e., to alkylating agents), prognostication, and/or

294 stratification in clinical trials ${ }^{25-27}$. 
295 The rationale for the use of TMZ in PC is supported by several clinical and pharmacological 296 studies in neuroendocrine and primary brain tumors, indicating its excellent blood-brain barrier 297 penetration, in addition to its homogenous distribution in CNS and extra-CNS tissues. ${ }^{12,28}$

298 Ramanathan et al. reported an objective response rate of $34 \%$ in a phase II study using 299 dacarbazine (DTIC) intravenously as single-agent therapy for metastatic pancreatic 300 neuroendocrine tumors (PNETs). ${ }^{29} \mathrm{TMZ}$, which is bioactivated into the same metabolite as DTIC 301 (5-methyltriazenoimidazole-4-carboxamide, MTIC), has the advantage of undergoing 302 spontaneously decarboxylation (and therefore bypassing hepatic activation) ${ }^{30}$. The relatively 303 mild toxicity profile and ease of use (oral formulation) are also advantages of TMZ over other 304 chemotherapies. TMZ works by inducing cell apoptosis or cell senescence in rapidly dividing 305 cells and it is non-specific to any mitosis phase. Furthermore, TMZ demonstrated good response in primary brain tumors and previously untreated brain metastases when added to 307 radiotherapy. ${ }^{31}$ In parallel, the relatively low MGMT expression seen in NET, including PA and 308 PC, increases their sensitivity to alkylating agents such as TMZ. ${ }^{32,33}$ The 2017 European guideline 309 for pituitary tumors encourages testing of MGMT expression by IHC on aggressive pituitary 310 tumors and pituitary carcinomas (even though only a minority of them exhibit homogenous 311 MGMT expression), as it may help predict treatment success with temozolomide, the first-line 312 chemotherapy recommended for such tumors. ${ }^{22,34}$ Nevertheless, MGMT may not be the sole 313 driver for susceptibility to TMZ, and other enzymes, such as MSH6, may also contribute to PC 314 sensitivity to alkylating agents. ${ }^{25}$ For this reason, caution is warranted when using MGMT 315 expression as the only criterion to decide on the use of temozolomide in PC and locally 
317 in patients with high expression have been described. ${ }^{35}$ Therefore, a trial of therapy with TMZ

318 may be warranted regardless of MGMT status, particularly if other treatment options have

319 been exhausted.

320 Further reports described the effective use of TMZ combined with capecitabine in recurrent

321 and metastatic PNETs ${ }^{11,12,21,36-38}$. The addition of capecitabine to TMZ (CAPTEM) is based on the

322 theory that sequential pretreatment with capecitabine may potentiate the cytotoxicity of TMZ

323 by synergistically depleting thymidine, leading to apoptosis. ${ }^{11,28}$ A recent randomized Phase II

324 study has shown improved PFS and OS of capecitabine in combination with TMZ compared to

325 TMZ alone in advanced pancreatic NETs; ${ }^{39}$ however, this has not been tested in PCs and

326 whether CAPTEM is superior to TMZ alone in this patient population remains unknown.

327 Potential benefits of using combined therapy must be carefully weighed against the potential

328 for added toxicities. In our series, the use of CAPTEM therapy in patients with previous

329 progression after TMZ monotherapy was limited to 3 cases, and definitive conclusions about

330 the benefit of CAPTEM in the setting of TMZ failure cannot be drawn.

331 In our patient cohort, we observed a longer median time between PC diagnosis and first PC

332 recurrence in patients treated with upfront TMZ-based therapy (30 months) compared with

333 patients treated with other lines of upfront therapy that was not TMZ-based (10 months),

334 although the low number of patients and the potential bias inherent in retrospective series

335 limits generalization of the validity of these results. Our results are also superior to those

336 previously reported in the literature (Table 2); this includes results from a contemporary study

337 in which TMZ was commonly prescribed, and which reported that $42.5 \%$ of PC patients were

338 deceased with median follow-up of 12 months. ${ }^{40}$ Nevertheless, we are unable to confirm 
whether the favorable outcome seen with TMZ is due to "first therapy effect", when the longest PFS is usually seen, whether it is related to higher TMZ efficacy or, more likely, whether

341 it is a combination of both.

342 Radiotherapy with concurrent TMZ was completed in 3 patients, which was then followed by

343 cisplatin $(n=1), T M Z(n=1)$ and capecitabine plus TMZ $(n=1)$. Two of these patients had disease

344 control for more than 2 years, and all 3 patients survived for at least 3 years. This is in

345 agreement with a recent European survey which showed that concurrent TMZ and RT was

346 associated with increased response rate compared to $T M Z$ alone. ${ }^{40}$ The longer survival rates in

347 our cohort may be correlated to the multimodality approach and/or TMZ use, especially

348 considering that all long-term ( $>5$ years) survivors were treated with TMZ-based therapy.

349 This study has the limitations typical of an observational retrospective study, including the small 350 cohort and single-center setting. Therefore, we are unable to draw any comparative statistical 351 analysis among the different treatment modalities given to each PC patient or across PC

352 patients. Furthermore, a tertiary institution serving as a national and international referral 353 center invariably has a selection bias for patients with more severe conditions and poorer 354 prognosis. Finally, the confounding bias related to several lines of therapy in a relatively short 355 timeframe also limits the interpretation of a true cause-effect phenomenon between each line 356 of therapy and the observed outcome. Despite all these limitations, our series provides valuable 357 information on the demographics, tumor characteristics, treatment modalities and outcome of 358 patients with this rare malignancy for which prospective data are lacking to guide estimation of prognosis and decisions on therapy. 


\section{Conclusion:}

363 PC is a rare and aggressive neuroendocrine malignancy. Local recurrence is frequent and

364 metastases occur in both CNS as well as extra-CNS locations. A combination of surgical

365 resection, radiotherapy, and chemotherapy (particularly TMZ-based) may result in prolonged

366 survival. In this case series, which is the largest single-institution experience published in the

367 literature, early use of chemotherapy, specifically TMZ, combined with standard PC

368 management (surgical resection and radiotherapy) was well tolerated and associated with

369 improved survival rates compared to the previous literature.

\section{References}

373 1. Heaney AP. Clinical review: Pituitary carcinoma: difficult diagnosis and treatment. J Clin

$374 \quad$ Endocrinol Metab. 2011;96(12):3649-3660.

375 2. Doniach I. Pituitary carcinoma. Clin Endocrinol (Oxf). 1992;37(2):194-195.

376 3. Scheithauer BW, Kovacs KT, Laws ER, Jr., Randall RV. Pathology of invasive pituitary tumors with 377 special reference to functional classification. J Neurosurg. 1986;65(6):733-744.

378 4. Lopes MBS. The 2017 World Health Organization classification of tumors of the pituitary gland: a summary. Acta Neuropathol. 2017;134(4):521-535. 
5. Mete O, Ezzat S, Asa SL. Biomarkers of aggressive pituitary adenomas. J Mol Endocrinol. 2012;49(2):R69-78.

382 6. Kaltsas GA, Nomikos P, Kontogeorgos G, Buchfelder M, Grossman AB. Clinical review: Diagnosis and management of pituitary carcinomas. J Clin Endocrinol Metab. 2005;90(5):3089-3099.

7. Pernicone PJ, Scheithauer BW, Sebo TJ, et al. Pituitary carcinoma: a clinicopathologic study of 15 cases. Cancer. 1997;79(4):804-812.

8. Morokuma H, Ando T, Hayashida T, et al. A case of nonfunctioning pituitary carcinoma that responded to temozolomide treatment. Case Rep Endocrinol. 2012;2012:645914.

9. Losa M, Bogazzi F, Cannavo S, et al. Temozolomide therapy in patients with aggressive pituitary adenomas or carcinomas. J Neurooncol. 2016;126(3):519-525.

10. Fadul CE, Kominsky AL, Meyer LP, et al. Long-term response of pituitary carcinoma to temozolomide. Report of two cases. J Neurosurg. 2006;105(4):621-626.

392 11. Thearle MS, Freda PU, Bruce JN, Isaacson SR, Lee Y, Fine RL. Temozolomide (Temodar(R)) and capecitabine (Xeloda(R)) treatment of an aggressive corticotroph pituitary tumor. Pituitary. 2011;14(4):418-424.

12. Fine RL, Gulati AP, Krantz BA, et al. Capecitabine and temozolomide (CAPTEM) for metastatic, well-differentiated neuroendocrine cancers: The Pancreas Center at Columbia University experience. Cancer Chemother Pharmacol. 2013;71(3):663-670.

398 13. Lim S, Shahinian H, Maya MM, Yong W, Heaney AP. Temozolomide: a novel treatment for pituitary carcinoma. Lancet Oncol. 2006;7(6):518-520.

400 14. Campdera M, Palacios N, Aller J, et al. Temozolomide for aggressive ACTH pituitary tumors: 401 failure of a second course of treatment. Pituitary. 2016;19(2):158-166.

402 15. Ceccato F, Lombardi G, Manara R, et al. Temozolomide and pasireotide treatment for aggressive pituitary adenoma: expertise at a tertiary care center. J Neurooncol. 2015;122(1):189-196. 
404 16. Strowd RE, Salvatori R, Laterra JJ. Temozolomide retreatment in a recurrent prolactin-secreting

405

406

407

408

409

410

411

412

413

414

415

416

417

418

419

420

421

422

423

424

pituitary adenoma: Hormonal and radiographic response. J Oncol Pharm Pract. 2016;22(3):517-

522.

17. Bruno OD, Juarez-Allen L, Christiansen SB, Danilowicz K. Long-lasting complete remission after therapy with temozolomide in two patients with macrocorticotropinoma causing Cushing's disease. Clin Endocrinol (Oxf). 2015;83(1):143-145.

18. Bengtsson D, Schroder HD, Andersen M, et al. Long-term outcome and MGMT as a predictive marker in 24 patients with atypical pituitary adenomas and pituitary carcinomas given treatment with temozolomide. J Clin Endocrinol Metab. 2015;100(4):1689-1698.

19. Gulati AP, Krantz B, Moss RA, et al. Treatment of multiple endocrine neoplasia 1/2 tumors: case report and review of the literature. Oncology. 2013;84(3):127-134.

20. Zhong C, Yin S, Zhou P, Jiang S. Pituitary atypical adenoma or carcinoma sensitive to temozolomide combined with radiation therapy: a case report of early identification and management. Turk Neurosurg. 2014;24(6):963-966.

21. Kamiya-Matsuoka C, Cachia D, Waguespack SG, et al. Radiotherapy with concurrent temozolomide for the management of extraneural metastases in pituitary carcinoma. Pituitary. 2016;19(4):415-421.

22. Raverot G, Burman P, McCormack A, et al. European Society of Endocrinology Clinical Practice Guidelines for the management of aggressive pituitary tumours and carcinomas. Eur J Endocrinol. 2018;178(1):G1-G24.

23. Lloyd RV OR, Klöppel G \& Rosai J. Pathology and Genetics of Tumours of Endocrine Organs. In: press I, ed. IARC WHO Classification of Tumours.2017. 
24. AbdelBaki MS, Waguespack SG, Salceda V, et al. Significant response of pituitary carcinoma to carboplatin, leucovorin and fluorouracil chemotherapy: a pediatric case report and review of the literature. J Neurooncol. 2017;135(1):213-215.

25. Matsuno A, Murakami M, Hoya K, et al. Molecular status of pituitary carcinoma and atypical adenoma that contributes the effectiveness of temozolomide. Med Mol Morphol. 2014;47(1):17.

26. Hirohata T, Asano K, Ogawa Y, et al. DNA mismatch repair protein (MSH6) correlated with the responses of atypical pituitary adenomas and pituitary carcinomas to temozolomide: the national cooperative study by the Japan Society for Hypothalamic and Pituitary Tumors. J Clin Endocrinol Metab. 2013;98(3):1130-1136.

27. Lau Q, Scheithauer B, Kovacs K, Horvath E, Syro LV, Lloyd R. MGMT immunoexpression in aggressive pituitary adenoma and carcinoma. Pituitary. 2010;13(4):367-379.

28. Zacharia BE, Gulati AP, Bruce JN, et al. High response rates and prolonged survival in patients with corticotroph pituitary tumors and refractory Cushing disease from capecitabine and temozolomide (CAPTEM): a case series. Neurosurgery. 2014;74(4):E447-455; discussion E455.

29. Ramanathan RK, Cnaan A, Hahn RG, Carbone PP, Haller DG. Phase II trial of dacarbazine (DTIC) in advanced pancreatic islet cell carcinoma. Study of the Eastern Cooperative Oncology GroupE6282. Ann Oncol. 2001;12(8):1139-1143.

30. Carmen Avendano JCM. DNA Alkylating Agents. In: Carmen Avendano JCM, ed. Medicinal Chemistry of Anticancer Drugs. Elsevier; 2008:139-176.

31. Antonadou D, Paraskevaidis M, Sarris G, et al. Phase II randomized trial of temozolomide and concurrent radiotherapy in patients with brain metastases. J Clin Oncol. 2002;20(17):3644-3650. 
32. Kulke $\mathrm{MH}$, Hornick JL, Frauenhoffer $\mathrm{C}$, et al. O6-methylguanine DNA methyltransferase deficiency and response to temozolomide-based therapy in patients with neuroendocrine tumors. Clin Cancer Res. 2009;15(1):338-345.

33. Salehi F, Scheithauer BW, Kovacs K, et al. O-6-methylguanine-DNA methyltransferase (MGMT) immunohistochemical expression in pituitary corticotroph adenomas. Neurosurgery. 2012;70(2):491-496; discussion 496.

34. Salehi F, Scheithauer BW, Kros JM, et al. MGMT promoter methylation and immunoexpression in aggressive pituitary adenomas and carcinomas. J Neurooncol. 2011;104(3):647-657.

35. Ortiz LD, Syro LV, Scheithauer BW, et al. Temozolomide in aggressive pituitary adenomas and carcinomas. Clinics (Sao Paulo). 2012;67 Suppl 1:119-123.

36. Devata S, Kim EJ. Neoadjuvant chemotherapy with capecitabine and temozolomide for unresectable pancreatic neuroendocrine tumor. Case Rep Oncol. 2012;5(3):622-626.

37. Saranga-Perry V, Morse B, Centeno B, Kvols L, Strosberg J. Treatment of metastatic neuroendocrine tumors of the thymus with capecitabine and temozolomide: a case series. Neuroendocrinology. 2013;97(4):318-321.

38. Crespo G, Jimenez-Fonseca P, Custodio A, et al. Capecitabine and temozolomide in grade 1/2 neuroendocrine tumors: a Spanish multicenter experience. Future Oncol. 2017;13(7):615-624.

39. Kunz PL CP, Nimeiri H, Fisher GA, Longacre TA, Suarez CJ, Yao JC, Kulke MH, Hendifar AE, Shanks JC, Shah MH, Zalupski M, Schmulbach EL, Reidy DL, Strosberg JR, O'Dwyer PJ, Benson AB. A randomized study of temozolomide or temozolomide and capecitabine in patients with advanced pancreatic neuroendocrine tumors: A trial of the ECOG-ACRIN Cancer Research Group (E2211). J Clin Oncol. 2018;36(suppl). 
470

40. McCormack A, Dekkers OM, Petersenn S, et al. Treatment of aggressive pituitary tumours and carcinomas: results of a European Society of Endocrinology (ESE) survey 2016. Eur J Endocrinol. 2018;178(3):265-276.

41. Kaltsas GA, Mukherjee JJ, Plowman PN, Monson JP, Grossman AB, Besser GM. The role of cytotoxic chemotherapy in the management of aggressive and malignant pituitary tumors. J Clin Endocrinol Metab. 1998;83(12):4233-4238.

42. Roncaroli F, Nose V, Scheithauer BW, et al. Gonadotropic pituitary carcinoma: HER-2/neu expression and gene amplification. Report of two cases. J Neurosurg. 2003;99(2):402-408.

43. Raverot G, Sturm N, de Fraipont F, et al. Temozolomide treatment in aggressive pituitary tumors and pituitary carcinomas: a French multicenter experience. J Clin Endocrinol Metab. 2010;95(10):4592-4599.

44. Hansen TM, Batra S, Lim M, et al. Invasive adenoma and pituitary carcinoma: a SEER database analysis. Neurosurg Rev. 2014;37(2):279-285; discussion 285-276.

45. Wang YQ, Fan T, Zhao XG, Liang C, Qi XL, Li JY. Pituitary carcinoma with intraspinal metastasis: report of two cases and review of the literature. Int J Clin Exp Pathol. 2015;8(8):9712-9717.

46. Yoo F, Kuan EC, Heaney AP, Bergsneider M, Wang MB. Corticotrophic pituitary carcinoma with cervical metastases: case series and literature review. Pituitary. 2018;21(3):290-301. 
Table 1

\begin{tabular}{|c|c|c|c|c|c|c|c|c|c|c|}
\hline $\begin{array}{c}\text { PC } \\
\text { subtype }\end{array}$ & $\begin{array}{l}\text { Age (v), } \\
\text { Gender }\end{array}$ & $\begin{array}{l}\text { PA to PC } \\
\text { latency } \\
\text { (v) }\end{array}$ & $\begin{array}{l}\text { Therapy upon } \\
\text { PC diagnosis }\end{array}$ & $\begin{array}{c}\text { PC } \\
\text { recurrences }\end{array}$ & $\begin{array}{l}\text { Therapy upon } \\
\text { PC recurrence }\end{array}$ & $\begin{array}{c}\text { CNS } \\
\text { metastasis }\end{array}$ & $\begin{array}{l}\text { Extra-CNS } \\
\text { metastasis }\end{array}$ & Current status & $\begin{array}{l}\text { Longest } \\
\text { PFS (v) }\end{array}$ & OS $(y)$ \\
\hline NC & $46, M$ & 8 & $\mathrm{TMZ}^{*}$ & 1 & - & dura, LMD & - & deceased & 2.5 & 3 \\
\hline NC & $44, F$ & 1 & $\mathrm{RT}+\mathrm{TMZ} \rightarrow$ cispl $^{*}$ & 0 & - & & - & surveillance & $2.5+$ & $3+$ \\
\hline NC & $69, \mathrm{M}$ & 9 & $\mathrm{TMZ}^{*}$ & 1 & CSRT & dura & bone & beva & 3.5 & $4+$ \\
\hline NC+ & $69, \mathrm{~F}$ & 15 & $\mathrm{RT}+\mathrm{TMZ}^{\prime} \rightarrow \mathrm{CAPTEM}^{*}$ & 0 & - & - & lymph node, bone & surveillance & $3.5+$ & $4+$ \\
\hline NC & $36, M$ & 11 & none* & 4 & $T M Z$ & dura & lung, bone & $T M Z$ & 13 & $13.5+$ \\
\hline ACTH & $45, M$ & 2 & RT $\rightarrow$ cisol+etoo $0^{*}$ & 0 & - & - & liver & deceased & $<0.5$ & $<1$ \\
\hline АСТН & $54, \mathrm{~F}$ & 29 & $\mathrm{RT} \rightarrow \mathrm{TMZ}^{*}$ & 0 & - & dura, LMD & - & surveillance & $2+$ & $2+$ \\
\hline $\mathrm{ACTH}+$ & $41, M$ & 4 & RT & 10 & $\begin{array}{l}\text { TMZ*, CAPTEM, } \\
\text { CCNU+bev }\end{array}$ & dura & $\begin{array}{l}\text { liver, orbits, } \\
\text { lymph node }\end{array}$ & immunotherapy & 1.5 & $5.5+$ \\
\hline АСTH & $30, \mathrm{~F}$ & 11 & RT & 4 & SRS, TMZ* & dura, LMD & bone & TMZ re-challenge & $3+$ & $6+$ \\
\hline $\mathrm{ACTH}+$ & $16, F$ & 5 & RT & 3 & CAPTEM, carbo-5FU* & dura & - & surveillance & 2 & $10.5+$ \\
\hline PRL & $81, F$ & 12 & RT & 2 & TMZ* & dura & lung & deceased & $<1$ & $<1$ \\
\hline PRL & $47, M$ & 2 & none & 0 & - & dura & - & deceased & $<0.5$ & $<1$ \\
\hline PRL & $25, M$ & 2 & $\mathrm{RT} \rightarrow \mathrm{TMZ}^{*}$ & 0 & carbo+pacl, CAPTEM & dura & bone, liver & deceased & 2.5 & 10.5 \\
\hline PRL+ & $57, \mathrm{~F}$ & 6 & cispl+etop & 4 & TMZ*, CAPTEM, PD-1 & - & bone, liver & immunotherapy & 1.5 & $2.5+$ \\
\hline LH/FSH & $23, \mathrm{~F}$ & 2 & CYVADIC* & 1 & CWADIC & dura & - & deceased & $<1$ & 1.5 \\
\hline LH/FSH & $46, M$ & 4 & $\mathrm{TMZ}^{*}$ & 4 & XRT+TMZ, RT & $\begin{array}{l}\text { epidural } \\
\text { spine }\end{array}$ & - & deceased & $\ll 1$ & 5 \\
\hline GH & $23, M$ & 2 & $\mathrm{TMZ}^{*}$ & 0 & - & dura & - & TMZ & $<0.5+$ & $<1+$ \\
\hline
\end{tabular}

Table 1: PC, pituitary carcinoma; NC, null cell; ACTH/PRL/LH/FSH/GH, hormone-secreting PC; PA,

500 pituitary adenoma; SRS, stereotactic radiosurgery; RT+TMZ, concurrent chemoradiation; RT, photon

501 radiotherapy; PRT, proton radiotherapy; TMZ, temozolomide; cispl, cisplatin; CAPTEM,

502 capecitabine+temozolomide; etop, etoposide; carbo, carboplatin; 5FU, 5-fluorouracil; PD-1, PD-1

503 inhibitor; pacl, paclitaxel; vinc, vincristine; CyVADIC, cyclophosphamide+vincristine+doxorubicin+

504 dacarbazine; bev, bevacizumab; CSRT, craniospinal radiotherapy; LMD, leptomeningeal disease; *,

505 therapy prior to longest PFS; + , previously published cases from MDACC in the literature. 
Table 2

\begin{tabular}{|c|c|c|c|c|c|c|c|c|}
\hline Study & $\begin{array}{l}\text { Study } \\
\text { period }\end{array}$ & $\begin{array}{l}\text { Single } \\
\text { center }\end{array}$ & $\begin{array}{l}\text { PC } \\
\text { cases }\end{array}$ & PC type & $\begin{array}{l}\text { TMZ } \\
\text { Tx }\end{array}$ & $\begin{array}{l}\text { other Tx } \\
\text { modalities }\end{array}$ & $\mathrm{PFS}^{*}(\mathrm{mo})$ & $\mathrm{OS}^{*}(\mathrm{mo})$ \\
\hline $\begin{array}{l}\text { Pernicone, } \\
1997^{7}\end{array}$ & $1955-1994$ & Yes & 15 & $\operatorname{ACTH}(7), \operatorname{PRL}(7), N C(1)$ & 0 & $\begin{array}{l}\text { surgery, RT, } \\
\text { cytotoxic agents }\end{array}$ & Not reported & 1 -у OS $66 \%$, mOS 22 \\
\hline $\begin{array}{l}\text { Kaltsas, } \\
1998^{41}\end{array}$ & 1970-1997 & Yes & 4 & ACTH(1), PRL(3) & 0 & $\begin{array}{c}\text { cytotoxic agents, } \\
\text { RT }\end{array}$ & 11.5 & 11.5 \\
\hline $\begin{array}{l}\text { Roncaroli, } \\
2003^{42}\end{array}$ & $1983-2013$ & Yes & 2 & PRL(2) & & cytotoxic agents & 9 & 10 \\
\hline $\begin{array}{l}\text { Raverot, } \\
2010^{43}\end{array}$ & $\begin{array}{l}\text { Not } \\
\text { reported }\end{array}$ & No & 5 & ACTH(2), PRL(3) & 5 & surgery, RT & Not reported & Not reported \\
\hline $\begin{array}{l}\text { Hirohata, } \\
2013^{26}\end{array}$ & $2006-2011$ & No & 12 & $\begin{array}{c}\mathrm{ACTH}(3), \mathrm{PRL}(3), \mathrm{NC}(4), \\
\mathrm{NOS}(2)\end{array}$ & 10 & Not reported & Not reported & Not reported \\
\hline $\begin{array}{l}\text { Hansen, } \\
2014^{44}\end{array}$ & $1973-2008$ & No & 7 & Not reported & 0 & surgery & Not reported & $\begin{array}{l}1-y \text { OS } 57.1 \%, 2-y \text { OS } \\
28.6 \%, 5-y \text { OS } 28.5 \%\end{array}$ \\
\hline $\begin{array}{l}\text { Bengtsson, } \\
2015^{18}\end{array}$ & $\begin{array}{l}\text { Not } \\
\text { reported }\end{array}$ & No & 8 & $\operatorname{ACTH}(3), \operatorname{PRL}(2), G H(3)$ & 8 & $\begin{array}{l}\text { surgery, } \mathrm{RT}, \\
\text { cytotoxic agents }\end{array}$ & Not reported & Not reported \\
\hline $\begin{array}{l}\text { Wang, } \\
2015^{45}\end{array}$ & 2006-2015 & Yes & 2 & $\mathrm{ACTH}(1), \mathrm{GH}(1)$ & 0 & surgery, RT & $\begin{array}{c}156(\mathrm{ACTH}) \\
22(\mathrm{GH})\end{array}$ & $\begin{array}{c}178(\mathrm{ACTH}) \\
34(\mathrm{GH})\end{array}$ \\
\hline $\begin{array}{l}\text { Yoo, } \\
2018^{46}\end{array}$ & 2018 & & 2 & $\mathrm{ACTH}(2)$ & 2 & surgery, RT & 6 & $12+$ \\
\hline $\begin{array}{c}\text { Santos- } \\
\text { Pinheiro, } \\
2018\end{array}$ & $1994-2017$ & Yes & 17 & $\begin{array}{c}\mathrm{ACTH}(5), \mathrm{PRL}(4), \mathrm{GH}(1), \\
\mathrm{FSH} / \mathrm{LH}(2), \mathrm{NC}(5)\end{array}$ & 15 & surgery, RT & 18 & $\begin{array}{c}\operatorname{mOS} 36 ; 2-y \text { OS } 65 \%, 3- \\
\text { y OS } 53 \%, 5-y \text { OS } 29 \%\end{array}$ \\
\hline
\end{tabular}

514 Table 2: Previously published pituitary carcinoma case series by single and multicenter groups. *:

515 median PFS and OS since PC diagnosis. Abbreviations: PC, pituitary carcinoma; TMZ, temozolomide; PFS,

516 progression-free survival; $\mathrm{O}$, overall survival; $\mathrm{ACTH}$, adrenocorticotrophic hormone; PRL, prolactin

517 hormone; NC, null-cell; GH, growth hormone; FSH/LH, follicle-stimulating hormone/luteinizing hormone;

518 NOS, not otherwise specified; RT, radiotherapy; 


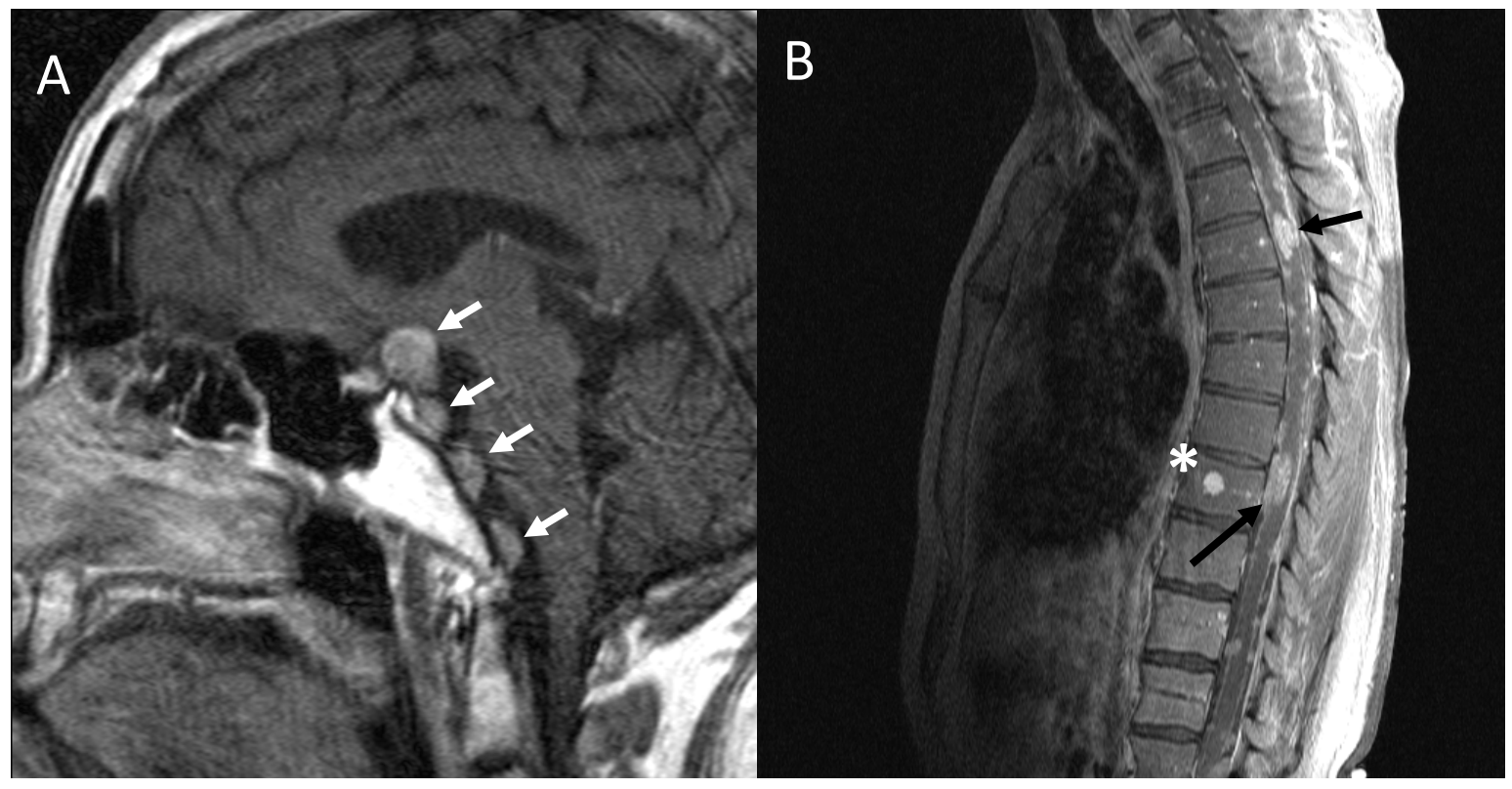

537 Figure 1. A) 17 year old girl with atypical pituitary adenoma, previously treated with surgery and

538 radiation, presented with Cushing syndrome. Sagittal T1 post-contrast image shows multiple dural-

539 based metastases (white arrows). B) 48 year old man with surgically proven dural-based metastases

540 from pituitary carcinoma was treated with multiple surgeries, radiation and chemotherapy. He

541 presented with foot drop. Sagittal T1 post-contrast fat-saturated image shows presumed bone

542 metastasis $(*)$ and innumerable intrathecal metastases (black arrows). 